\title{
Sobre a representação mecânica das forças elétrica, magnética e galvânica, de William Thomson: uma leitura comentada
}

On a Mechanical Representation of Electric, Magnetic and Galvanic Forces, by William Thomson: a commented reading

\author{
M. C. de Lima*, R. S. Costa
}

Faculdade de Física, Universidade Federal do Pará, Belém, PA, Brasil

Recebido em 18 de Setembro, 2016. Revisado em 09 de Dezembro, 2016. Aceito em 16 de Dezembro, 2016.

Apresentamos e comentamos o artigo homônimo de William Thomson, originalmente publicado em 1847, no qual o autor explora a analogia entre os estados de deformação em um sólido elástico e as configurações das linhas de força de Faraday. Nele Thomson introduz pela primeira vez o potencial vetorial magnético, associando-o à deformação rotacional de um sólido. Apresentamos também um apanhado histórico do contexto dentro do qual o original de Thomson foi concebido.

Palavras-chave: Sólido elástico, éter luminífero, linha de força, análogo mecânico.

We present and comment the homonimous article originally published by William Thomson, in 1847, in which the author explores the analogy between the estates of deformation in an elastic solid and the configurations of Faraday's lines of force. In this article Thomson has introduced for the first time the magnetic potential vector, associated in this context with rotational deformations in the solid. We present as well, the historical context in which Thomson's original paper has been conceived.

Keywords: elastic solid, luminiferous aether, line of force, mechanical analogue.

\section{Introdução}

Nos anos de 1840, o jovem William Thomson (futuro Lord Kelvin), aos 21 anos, daria início a um programa de formalização teórica alternativa da ciência elétrica e magnética, o qual considerava ser baseado nas ideias de Michael Faraday.

Faraday, aos 49 anos, retomava suas atividades científicas, após o colapso nervoso de 1839. Imortalizara seu nome na ciência britânica, e fora dela, após a descoberta da indução eletromagnética (1831), o estabelecimento das leis da eletrólise (1833), a descoberta da capacidade indutiva dos dielétricos (1837), entre tantos outros feitos [1]. Em sua obra eletromagnética Faraday concebia que as ações entre imãs, correntes, etc. ocorriam ao longo de linhas curvas e que tais ações resultavam de um estado de tensão no espaço. A transmissão da força era vista como ocorrendo de forma contígua, ponto a ponto do espaço, alterando continuamente a configuração deste estado de tensão, o campo. Este estado era, por ele, visualizado através de sua noção de linhas de força. Dada a formação auto-didata e heterodoxa de Faraday, suas concepções não encontravam ressonância nas formulações teóricas, então vigentes, acerca dos fenômenos elétricos e magnéticos.

Fazer uma ponte entre as concepções de Faraday e a física-matemática era a tarefa na qual Thomson estava decidido a engajar-se. Ao traçar os meios de como construí-la lançou as linhas mestras que seriam trilhadas por James Clerk Maxwell, George Francis FitzGerald, Oliver Heaviside, John Henry Poynting, Joseph Larmor e Hendrik Antoon Lorentz, e que, conjuntamente com as descobertas de Heinrich Rudolf Hertz, iriam nos legar o que hoje chamamos de eletromagnetismo clássico.

*Endereço de correspondência: mclima@ufpa.br. 


\section{Belfast, Glasgow, Cambridge e Paris}

Thomson deixou sua Irlanda natal em 1834, seguindo inicialmente para Glasgow onde encontrou um ambiente acadêmico que, diferentemente das tradicionais universidades inglesas, valorizava fortemente a física-matemática francesa. Assim, cedo familiarizouse com a obra de Jean-Baptiste Joseph Fourier ("Théorie analytique de la Chaleur"), a qual lera aos 16 anos, Joseph-Louis Lagrange ("Mécanique Analytique”), Pierre Simon Laplace (Mécanique Céleste"), entre outros.

Nos seus primeiros anos na Universidade de Cambridge, logo publicou "On the uniform motion of heat and its connection with the mathematical theory of electricity" [2], no qual transpunha a teoria de Fourier, da propagação do calor em regime estacionário, para o contexto da eletrostática; fazendo o tipo de correspondência matemática que será uma característica de suas teorizações, nos diversos campos da física. Em Cambridge Thomson redescobriu o "Essay on the Application of Mathematical Analysis to the Theories of Electricity and Magnetism", de George Green, originalmente escrito em 1828, que exerceria grande influência nas diretrizes de suas investigações teóricas. Disputou o Mathematical Tripos de 1845, alcançando a posição de "second wrangler" e exames adicionais o permitiram conquistar ainda o prêmio Smith, sendo eleito fellow na Peterhouse, em Cambridge.

Daí seguiu para Paris, completando sua formação entre os franceses, onde trabalhou com Henri Victor Regnault, também interagindo com Jean-Baptiste Biot, Augustin-Louis Cauchy e Joseph Liouville. Liouville lhe chamou atenção sobre o potencial campo de investigação decorrente do aparente conflito entre as concepções de Faraday, em eletricidade, baseada na ideia de uma interação contígua, e as concepções da escola francesa, devidas a Charles Augustin de Coulomb e Siméon Denis Poisson, em que se assumia a ação instantânea à distância entre as supostas partículas constituintes do chamado fluido elétrico. Ao convencer-se da precisão matemática das ideias de Faraday, vislumbrou a possibilidade de com elas construir um cenário explicativo alternativo para os fenômenos eletrostáticos, eletrodinâmicos (galvânicos) e eletromagnéticos; então vistos como descritos satisfatoriamente pela ação à distância.

Sua aproximação com Faraday deu-se em 1845, quando mostrou a compatibilidade entre a lei de Coulomb e a descoberta de Faraday de que a in- teração entre corpos eletrizados dependia da natureza do meio dielétrico envolvente. No artigo "On the mathematical theory of electricity in equilibrium" 3 estabeleceu o tratamento matemático da influência dos meios polarizados, em harmonia com as ideias de Faraday [4]. Em carta de 6 de agosto de 1845 Thomson informou Faraday do artigo, quando então o sondou sobre a possibilidade de demonstrar a ação de um dielétrico polarizado, transparente, sobre a propagação da luz; fenômeno este que Thomson especulou dever existir [5]. Faraday já buscara em vão o fenômeno, mas, após ser instigado por Thomson, foi levado a experimentar se haveria a ação da magnetização em um meio transparente, sobre a propagação da luz. Em novembro daquele ano Faraday anunciou à Royal Society sua descoberta do efeito magneto-óptico, o efeito Faraday, da ação rotacional do campo magnético sobre o plano de polarização da luz polarizada [6], vinte anos antes que Maxwell propusesse sua teoria eletromagnética da luz.

A descoberta do efeito Faraday despertou em Thomson a intuição de se poder relacionar as linhas de força magnética às deformações rotacionais do meio etéreo. É neste contexto que Thomson publica, em 1847, este breve, porém significativo, "On a mechanical representation" ora traduzido. Nele Thomson não chega a sugerir que as ações que associamos ao campo eletromagnético sejam, a rigor, resultantes das deformações elásticas do éter sobre corpos ponderáveis. É sugestivo, porém, que a equação de equilíbrio para o meio elástico da qual ele partiu, equação (1), remetida a Stokes, tenha sido obtida por este último, no artigo referido por Thomson, com referência ao éter luminífero. É provável que Thomson já tivesse um plano mais ambicioso em mente, em 1847, conforme sugere sua obra eletromagnética subsequente. De fato, a partir dos anos de 1850, irá especular sobre uma teoria dinâmica para o meio etéreo, cujos aspectos diretamente observáveis seriam os fenômenos ópticos, eletromagnéticos, etc. [7. Se este caminho fecundo fosse trilhado até o fim, as linhas de força de Faraday talvez pudessem ser identificadas com linhas de deformação ou linhas de fluxo do meio etéreo subjacente.

$\mathrm{Na}$ sequência de suas investigações, Thomson abordará o efeito Faraday a partir de uma perspectiva dinâmica, em seu "Dynamical Illustations of the Magnetic and the Helicoidal rotatory effects on transparent Bodies on Polarized Light" [8], de 
1857, onde então as deformações rotacionais de "On a mechanical representations" darão lugar a vórtices moleculares do meio etéreo, como representação do campo magnético. Nisso inspirará o jovem Maxwell que adotará a concepção dos vórtices de Thomson, no seu On Physical Lines of Force [9], de 1862, onde Maxwell apresentará sua ideia seminal da corrente de deslocamento, como decorrência natural das propriedades elásticas dos vórtices moleculares, identificando o éter eletromagnético com o éter luminifero [10. Em 1865 Maxwell publicará seu "Dynamical Theory of Electromagnetic Field" [11], quando então procurará manter os elementos-chave de suas formulações teóricas, acerca do campo, visando preservar a ideia de que a luz é, afinal, um distúrbio eletromagnético, sem no entanto se comprometer fortemente com algum modelo particular para o meio etéreo. Propunha, assim, a teoria eletromagnética da luz e sustentava agora a existência da corrente de deslocamento, mesmo sem lhe atribuir uma fundamentação mecânica clara.

Thomson cultivou, até o fim de sua longa vida, uma atitude ambivalente em relação à corrente de deslocamento, descartando-a afinal como elo de ligação unificador entre os éteres luminífero e eletromagnético. Opôs-se à "assim chamada" teoria eletromagnética da luz, de Maxwell, não porque fosse avesso a ideia da unificação óptico-eletromagnética, da qual fora um precursor, mas por considerar o esquema maxwelliano reducionista $[12$. É preciso lembrar que o conceito de campo, como temos hoje, de um intermediador da interação desprovido de qualquer substrato, era uma noção insatisfatória por não fornecer o fundamento físico, isto é mecânico, da transmissão da força. Nesse sentido Thomson já foi apontado como um "mecanicista radical" 13. Os proponentes da teoria do campo julgavam, implícita ou explicitamente, estar contribuindo para a restauração de uma física na tradição de Descartes, onde não há lugar para o vazio no universo.

Quarenta anos à frente do momento em que Thomson publicou o artigo aqui traduzido, em 1888, Hertz revelou ao mundo as ondas de indução elétrica. Maxwell que falecera prematuramente em 1879 não testemunhou o fato. Nesta altura, as ondas de Hertz foram antes vistas como prova de que o mesmo meio etéreo era responsável pela propagação da luz e das ações eletromagnéticas, do que uma prova definitiva da teoria eletromagnética da luz de Maxwell. Conforme disse Thomson em 1893 no prefácio à edição inglesa da obra de Hertz [14:

"desde que Faraday ofendeu pela primeira vez os físicos matemáticos com suas linhas de força curvas, muitos trabalhadores e muitos pensadores ajudaram a construir no século dezenove a escola do plenum: um só éter para a luz, calor, eletricidade, magnetismo. E os [...] artigos elétricos de Hertz, trazidos ao mundo na última década do século, serão um monumento permanente da consumação esplêndida agora realizada."

\section{A ação à distância eletrostática e eletrodinâmica}

Até meados do século XIX os filósofos naturais "adequadamente letrados" haviam estabelecido uma abordagem da eletrostática e da magnetostática, a qual poderíamos chamar, por brevidade, de newtoniana. Nessa abordagem, que se tornou dominante, buscou-se o estabelecimento de "leis de força" que fossem centrais e dependentes das posições relativas de pares de objetos, cuja interação era regida pelo princípio da ação/reação. Os objetos por sua vez deviam ser dotados de alguma propriedade física que os tornava sensíveis à interação, à distância, no vazio. Assim foi que, no seio da sólida escola da física francesa, surgiu, em 1785, as "Memoire Sur L'Électricité et le Magnétisme" [15, de Coulomb, que inicialmente estabeleceu uma lei do inverso do quadrado da distância para a ação entre pólos magnéticos, $p$ e $p^{\prime}$, "isolados". Na sequência estabeleceu também a lei do inverso do quadrado para a interação eletrostática, entre cargas $e$ e $e^{\prime}$, ambas pensadas como centrais e de ação instantânea à distância. Em notação atual

$$
\vec{f}_{m}=\frac{p p^{\prime}}{r^{2}} \hat{r}, \quad \vec{f}_{e}=\frac{e e^{\prime}}{r^{2}} \hat{r} .
$$

Após a descoberta da ação da corrente elétrica sobre a agulha magnética, por Hans Christian Ørsted, em 1820, abriu-se um campo fértil de pesquisa para se explorar a unidade entre eletricidade e magnetismo. André-Marie Ampère, logo mostrou a ação magnética de uma corrente sobre outra, abandonando a visão de dois fluidos um elétrico outro magnético. Introduziu a terminologia eletrostática e eletrodinâmica, passando a encarar o magnetismo como um efeito secundário da eletricidade, quando em movimento. A culminância de suas investigações eletrodinâmicas surgiu em 1827, na "memoire" "Sur 
la théorie Mathématique des Phénomènes Életrodynamiques uniquement déduite de l'expérience" [16]. Nela Ampère estabeleceu os princípios matemáticos da eletrodinâmica, apresentando a expressão para a força entre dois elementos de corrente, $i d \vec{l} \mathrm{e}$ $i^{\prime} d \vec{l}^{\prime}$, central, instantânea à distância, obedecendo ao princípio da ação e reação em sua forma forte, a qual podemos escrever em notação atual como

$$
d \vec{f}_{A}=\frac{i i^{\prime}}{r^{2}}\left[3(\hat{r} \cdot d \vec{l})\left(\hat{r} \cdot d \vec{l}^{\prime}\right)-2\left(d \vec{l} \cdot d \vec{l}^{\prime}\right)\right] \hat{r}
$$

Com Ampère surgiu também a ideia de que o magnetismo dos imãs é, em última instância, um fenômeno eletrodinâmico.

A escola alemã avançou pelas mãos de Johann Carl Friedrich Gauss, Franz Neumann e Gustav Kirchhoff, entre outros; culminando com a primeira eletrodinâmica de partícula, nas mãos de Wilhelm Weber [17], em 1846. Na eletrodinâmica de Weber reunia-se, em uma só lei de força, os fenômenos da eletrostática, da eletrodinâmica e da indução eletromagnética, que fora descoberta por Faraday em 1831, supondo-se que resultavam da ação instantânea à distância e central entre elementos "atomísticos" de eletricidade, elementos esses que eram os constituintes do assim chamado, fluido elétrico. Usando uma notação vetorial anacrônica, a força de Weber era

$$
\vec{f}_{\mathrm{w}}=\frac{e e^{\prime}}{r^{2}}\left\{1-\frac{1}{W^{2}}\left(\dot{r}^{2}-2 r \ddot{r}\right)\right\} \hat{r},
$$

Nas expressões acima $\left(e, e^{\prime}\right)$ são as cargas elétricas, $r$ a separação instantânea entre elas e $(\dot{r}, \ddot{r})$ suas derivadas temporais. Na lei de Weber o termo independente do tempo reproduzia a lei de Coulomb, o termo em $\dot{r}$ permitia descrever a interação eletrodinâmica, reproduzindo a lei de Ampère, para a força eletrodinâmica, exibida acima (que não deve ser confundida com aquela que hoje é assim designada). Finalmente o termo em $\ddot{r}$ possibilitava explicar como uma corrente primária é capaz de induzir uma corrente secundária, isto é, permitia descrever a indução eletromgnética. A constante de Weber, $w$, dimensionalmente uma velocidade, fazia-se necessária para se poder compatibilizar os sistemas de unidades então chamados eletrostático e eletrodinâmico.

Tais resultados pareciam mostrar a adequação dos pré-supostos estabelecidos por Newton, para a interação gravitacional, também no contexto eletrostático, eletrodinâmico e consequentemente magnético.
A determinação do valor da constante $w$ se dará em 1856, pelo próprio Weber em colaboração com Rudolf Kohlrausch [18]. O valor encontrado, de

$$
W=4,394 \times 10^{8} \mathrm{~m} / \mathrm{s}
$$

chamará a atenção de vários pesquisadores, como Kirchhoff, Georg Friedrich Bernhard Riemann e Ludwig Valentin Lorenz, pela proximidade numérica com o valor conhecido da velocidade na luz no vácuo. De fato,

$$
\mathrm{w} / \sqrt{2}=3,1 \times 10^{8} \mathrm{~m} / \mathrm{s} .
$$

Mas será o jovem Maxwell, imerso num ambiente científico no qual a ideia da contiguidade da interação era cultivada, por seus antecessores Faraday e Thomson, que verá nisso a oportunidade para a construção de uma teoria eletromagnética da luz.

\section{O éter luminífero elástico}

As teorizações dos físicos não giravam apenas em torno das ações instantâneas à distância no vazio, embora essa tenha sido uma tendência no século XVIII. No domínio da óptica, porém, a concepção de que o universo é, afinal, totalmente preenchido por um meio sutil, o éter, se consolidava e iria se tornar uma concepção dominante na física até o fim do século XIX.

As contribuições pioneiras de Christiaan Huygens para a teoria ondulatória da luz, em seu "Traite de la Lumière", de 1690, quando então cunhou o termo éter luminífero, haviam sofrido um temporário ostracismo, em decorrência da predominância da hipótese corpuscular da luz, novamente associada a Newton. No ínicio do século XIX, porém, Thomas Young re-estabelecera o programa de uma teoria ondulatória para a luz. Em sua "Bakerian Lecture", lida perante a Royal Society em 24 de novembro de 1803, intitulada "Experiments and Calculations Relative to Physical Optics" [19] apresentou os comprimentos de onda associados à luz visível, entre 400 e 700 nanometros, obtidos em seus experimentos de interferência. Quando em 1817 Dominique Arago comunicou a Young que dois feixes de luz polarizados, de polarizações distintas, não sofriam interferência, Young sugeriu que a luz seria uma onda transversal, havendo um plano independente para cada modo de polarização independente. Tais experimentos, com luz polarizada, Arago fizera em parceria com Augustin-Jean Fresnel. 
Fresnel trabalhava em sua "Mémoire Sur la Diffraction de la lumière" [20, na qual discutia os fenômenos ópticos com base na hipótese ondulatória, e que venceu o prêmio da Academia Francesa de Ciências de 1819. Temos aí a primeira formulação matemática da teoria ondulatória da luz, havendo Fresnel colocado o princípio de Huygens em bases matemáticas precisas e, por isso, lembrado como princípio de Huygens-Fresnel. Em sua teoria da difração previu a presença de um ponto luminoso no centro da sombra geométrica de um disco (hoje lembrado como ponto de Arago). Poisson, que era um dos examinadores das memórias candidatas ao prêmio da academia, o havia previsto, usando a própria teoria de Fresnel, como uma espécie de "redução ao absurdo" (já que ele próprio defendia a teoria corpuscular). Disse que se renderia à teoria ondulatória se alguma experiência confirmasse a existência deste ponto, o que Fresnel e Arago foram capazes de demonstrar. Tais fatos fizeram de Young e Fresnel os re-fundadores da teoria ondulatória da luz.

Fresnel, diferentemente de Arago, aceitou a sugestão de Young acerca do caráter transversal da luz. Retomando a agenda de Huygens, no estudo da birrefringência, propôs, em 1821, o primeiro modelo de éter luminífero do tipo sólido elástico, de modo a suportar ondas transversais [21,22], isto é, ondas de cizalhamento. Convencidos de que a estrutura do éter luminífero não podia ser tão trivial, quanto Huygens supusera, e que a obra de Fresnell abria, assim, um novo campo de investigação, Green, Cauchy, James MacCullagh, George Gabriel Stokes e o próprio Thomson, tornaram-se, neste sentido, continuadores da obra de Young e Fresnel. Construíram o debate de como dotar o éter das propriedades mecânicas corretas, em consonância com a fenomenologia da óptica em meios homogêneos e isotrópicos, bem como nos meios cristalinos, onde surge anisotropia. Na teoria da reflexão, um dos problemas centrais que os assolava era o de eliminar o modo longitudinal das ondas de deformação, devido ao caráter puramente cizanhante da onda luminosa 23. Isto requeria que o éter luminífero fosse um estranho meio infinitamente mais resistente à compressão que à deformação. Outra característica dele, conforme apontada por Stokes [24], era que a sua rigidez somente se manifestaria quando fosse perturbado de forma brusca, como se supunha ser o caso dos distúrbios luminosos. Movimentos ordinários como o dos corpos celestes não provocariam sobre ele tais alterações bruscas de modo que tais corpos passavam através do éter sem percebê-lo.

Deste modo, à época em que Thomson publicou seu "On a mechanical representation", de 1847, aceitava-se que a luz era uma onda de deformação elástica, transversal, através do éter luminífero. Seria legítimo perguntar se o meio luminífero associava-se a outros fenômenos? Era natural responder que sim e, de fato, em 1851, o próprio Faraday, referindo-se à sua convicção de que a força magnética não era, afinal, uma ação à distância [25], diria:

"não é de todo improvável que se há um éter, ele deva ter outros usos além de simplesmente a transmissão de radiações".

\section{As linhas de força}

A série inaugural dos Experimental Researches in Electricity se deu com a descoberta da indução eletromagnética, em 1831, marcando o início da construção desta obra que Faraday somente consumará ao final de sua vida produtiva. Na sequência, na série VII dos Experimental Researches, publicada em 1834, iniciou seus estudos sobre a decomposição eletroquímica, que propôs chamar de eletrólise, os quais fora levado, em parte, pelo interesse em melhor compreender os mecanismos de geração da força eletromotriz pela pilha voltaica. Nele também começou a encarar o problema do entendimento da estrutura da matéria no nível fundamental, mostrando-se cético aos átomos de John Dalton e à hipótese do fluido elétrico como base do entendimento da eletricidade [23]. Nascia também aí, ainda que de forma reservada, sua oposição à ideia da ação à distância, em favor de uma ação contígua.

Os estudos da eletrólise levaram Faraday às suas investigações sobre a influência do meio dielétrico nos fenômenos de indução eletrostática, sendo o dielétrico pensado como um "quase eletrólito", que se polariza quando submetido à tensão externa sem propriemente sofrer a dissociação como no eletrólito. Daí surgir, na obra de Faraday, a concepção de que o estado elétrico de um dielétrico polarizado guardava alguma similaridade com o estado de deformação elástica em um sólido (o que para Thomson será um ponto de partida). A polarização era entendida, neste contexto, como aquilo que precede 
a corrente, sem propriamente se chegar ao limite em que esta última surge. É preciso lembrar que a ideia de polarização como o afastamento relativo dos centros de carga positivo e negativo, no interior da molécula, é anacrônica. Não havia, naquela altura, a aceitação hegemônica da existência de uma propriedade física fundamental da matéria chamada carga elétrica. Essa concepção de que um estado de polarização dielétrica é, na verdade, uma deformação elástica, será aceita por Maxwell quando da introdução da corrente de deslocamento, em 1862. A corrente elétrica por sua vez, era entendida por Faraday como uma sucessão de rupturas e religamentos da linha de força, estados de polarização sucessivamente rompidos e restabelecidos [26]. Em suas investigações sobre a indução em dielétricos mostrou, em 1838, que esses participavam ativamente no processo de indução eletrostática e introduziu a capacidade indutiva específica, a nossa constante dielétrica, característica do meio dielétrico que preenche o capacitor. Esta descoberta foi, para ele, uma prova indireta do caráter contíguo da interação elétrica, mostrando inclusive as linhas curvas que indicavam o "caminho" da força elétrica. Isto pareceu mostrar um conflito entre a ação elétrica instantânea à distância, cuja ação se dava em linha reta - conforme se admitia na lei de Coulomb - e a ação elétrica contígua, ao longo de linhas curvas, de Faraday. Para a comunidade científica, no entanto, essas eram opiniões heterodoxas acerca da natureza da ação elétrica e da corrente elétrica.

O colapso nervoso que se seguiu provocou certa descontinuidade das investigações de Faraday e talvez possamos dizer que o interesse de Thomson pelas concepções dele, a partir dos anos de 1840, pode ter sido um dos fatores que contribuíram para o reestabelecimento de suas atividades no campo da eletricidade e do magnetismo. De fato, um novo ciclo de descobertas fundamentais se deu quando Faraday revelou o efeito magneto-óptico 27] e mais, a existência dos diamagnéticos 28], conduzindo-o ao vislumbre de que, afinal, toda a matéria é magnética. Cada vez mais convencido do papel central desempenhado pela condição local da linha de força, na descrição das interações elétricas e magnéticas, Faraday ainda publicou em 1846 seu Thoughts on Rayvibrations [29], onde especulou se a luz e a radiação térmica não seriam meramente ondas resultantes das vibrações das linhas de força. Seria um vislumbre, uma intuição de que a luz seria uma onda eletro- magnética? Bem, poderíamos dizer que se tratava da uma especulação vaga, um palpite. No caso de Faraday porém, sabemos que sua intuição o guiou de forma certeira, no terreno da investigação experimental, às suas muitas descobertas.

Conforme já dissemos, Faraday não dispunha da formação matemática necessária para poder traduzir suas ideias em um sistema matemático formal. O jovem Thomson, era, por sua vez, uma pessoa qualificada para tal tarefa. Seu "On a mechanical representation", ora traduzido, é evidentemente uma iniciativa tímida frente ao programa geral de uma teoria dinâmica da ação contígua. Seu ponto de partida foi apenas o de explorar as eventuais analogias entre as linhas de força de Faraday e as linhas de deformação do meio etéreo. Efetivamente, ao enviar o artigo a Faraday, em carta datada de 11 de junho de 1847, ele dirá 5 :

"Meu caro senhor - envio-lhe em anexo o artigo de que lhe falei onde forneço uma analogia para as forças elétrica e magnética, por meio de deformações propagadas através de um sólido elástico. O que escrevi é simplesmente o esboço de uma analogia matemática. Não me aventurei sequer em sugerir tornála a fundação de uma teoria física da propagação das forças elétricas e magnéticas, a qual, se estabelecida, deveria fornecer como um resultado necessário a conexão entre forças elétricas e magnéticas, e mostrar como fenômenos magnéticos puramente estáticos podem se originar tanto da eletricidade em movimento, quanto de uma massa inerte tal qual um imã. Se tal teoria puder ser descoberta, ela também, muito provavelmente, explicaria o efeito do magnetismo sobre a luz polarizada, quando tomada em conexão com a teoria ondulatória da luz."

\section{Comentários sobre a tradução}

A notação original de Thomson é baseada nos componentes cartesianos de um vetor, tomados individualmente. Assim, por exemplo, o vetor deformação, o qual denotaríamos hoje por $\vec{u}$, foi denotado, no original, por suas projeções nos eixos como $(\alpha, \beta, \gamma)$. Seu divergente, $\nabla \cdot \vec{u}$, por

$$
\frac{d \alpha}{d x}+\frac{d \beta}{d y}+\frac{d \gamma}{d z}
$$

e assim por diante.

Embora a análise vetorial seja hoje a forma padrão de se expressar relações matemáticas na teoria ele- 
tromagnética, sabemos que mesmo após a sua formalização, com Gibbs e Heaviside, Thomson jamais lhe foi muito simpático. Em suas palavras [30]:

"Vetor é um sobrevivente inútil, ou desdobramento, dos quatérnions, e nunca teve a menor utilidade para qualquer criatura".

As notas de rodapé originais do próprio Thomson foram aqui colocadas nas referências e designadas por N.A. (notas do autor). Por N.T. indicamos as nossas notas de tradução. Nas notas explicativas (N.E.) fazemos então nossos comentários e lançamos mão, eventualmente, da correspondência entre expressões matemáticas da análise vetorial atual e as que Thomson nos apresenta, para ressaltar algum aspecto do que está sendo discutido, buscando melhor explicitar seu contexto.

Na seção seguinte (VII) apresentamos então a tradução comentada do artigo de William Thomson, cujo original pode ser encontrado em [31].

\section{Sobre a representação mecânica das forças elétrica, magnética e galvânica (Por William Thomson)}

Na série décima primeira do seu Experimental Researches in Electricity, o Sr. Faraday estabeleceu uma teoria da Indução Eletrostática na qual sugere a ideia de que à cada problema na teoria dos sólidos elásticos pode existir um problema, a ele relacionado, de distribuição de eletricidade em condutores, ou com as forças de atração e repulsão exercidas por corpos eletrizados. A chave para a representação das forças magnéticas e galvânicas foi proporcionada pela recente descoberta do Sr. Faraday, da ação dos sólidos transparentes sobre a luz polarizada quando sujeitos a forças magnéticas ou eletromagnéticas. Fui assim levado a encontrar três soluções particulares e distintas das equações de equilíbrio de um sólido elástico, das quais uma expressa o estado de deformação em que o deslocamento absoluto de uma partícula, em qualquer parte do sólido, representa a atração resultante, neste ponto, produzida por um corpo eletrizado. A outra fornece o estado de um sólido no qual cada elemento possui um deslocamento angular resultante definido, representando a força produzida por um corpo magnético, em direção e magnitude. A terceira representa, de forma similar, a força produzida por uma porção qualquer de um fio galvânico. Nestes últimos casos as direções das forças são dadas pelos eixos das rotações resultantes, impressas sobre os elementos do sólido.

As equações gerais do equilíbrio de um sólido elástico foram investigadas pelo Sr. Stokes, 11 sem qualquer pressuposto da relação entre a "compressibilidade cúbica" e a elasticidade referente à variações de forma, que não são acompanhadas por mudança de volume 2 Se denotarmos $\alpha, \beta, \gamma$ as projeções , sobre os três eixos de coordenadas retangulares, dos deslocamentos infinitesimais do ponto $(x, y, z)$ do sólido, segue, dos resultados do Sr. Stokes, que as equações de equilíbrio, quando o corpo está sujeito apenas a forças em suas superfícies limítrofes, podem ser escritas como segue 3

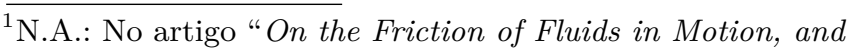
the Equilibrium and Motion of Elastic Solids", lido perante a Cambridge Philosophical Society, em 14 de Abril, 1845. Ver Trans. vol. VIII, Parte 3.

${ }^{2}$ N.E.: Thomson se refere ao módulo de compressibilidade $\mathrm{e}$ ao módulo de rigidez, respectivamente.

${ }^{3}$ N.E.: Podemos evitar os detalhes do artigo de Stokes e obter a referida condição de equilíbrio da teoria da elasticidade, tal como nos é apresentada hoje em Landau-Lifshitz, 32 ou Sokolnikoff, 33. A lei de Hooke é aí descrita como $\sigma_{i j}=2 \mu u_{i j}+(\kappa-2 \mu / 3) \nabla \cdot \vec{u} \delta_{i j}$, sendo $\sigma_{i j}$ o tensor de tensões, $u_{i j}=(1 / 2)\left(\partial_{j} u_{i}+\partial_{i} u_{j}\right)$ o tensor de deformações e $\nabla \cdot \vec{u}=u_{i}^{i}$ o seu traço. As constantes $(\kappa, \mu)$ são, respectivamente, o módulo de compressibilidade e o módulo de rigidez. As deformações rotacionais, por sua vez, são devidas ao tensor $\omega_{i j}=(1 / 2)\left(\partial_{j} u_{i}-\partial_{i} u_{j}\right)$, o qual se relaciona, por uma transformação de dualidade, ao rotacional do vetor deformação, isto é, $(\nabla \times \vec{u})^{k}=-\epsilon^{k i j} \omega_{i j}$. Tomando-se o traço na lei de Hooke decorre que $\sigma^{i}{ }_{i}=3 \kappa \nabla \cdot \vec{u}$. Sendo $p=-\sigma^{i}{ }_{i} / 3$ o análogo da pressão hidrostática em um fluido, virá então que $p=-\kappa \nabla \cdot \vec{u}$. As condições de equilíbrio em um sólido no qual não há forças de volume, mas apenas as de fronteira, são $\partial_{j} \sigma^{i j}=0$ e $\sigma_{i j}=\sigma_{j i}$. Dela resulta que $\mu \nabla^{2} \vec{u}+(\kappa+\mu / 3) \nabla(\nabla \cdot \vec{u})=0$, ou $\nabla^{2} \vec{u}+(\kappa+1 / 3) \nabla(\nabla \cdot \vec{u})=0$ se tomarmos o módulo de rigidez do meio como a unidade padrão $(\mu=1)$. Expressando em termos da pressão virá então $\nabla^{2} \vec{u}-\nabla p-(\nabla p) / 3 \kappa=0$. A condição proposta por Thomson resulta de tomarmos $\vec{u}=(\alpha, \beta, \gamma)$, o qual se assume incompressível $\nabla \cdot \vec{u}=0$, equação (2), sendo que $\kappa \rightarrow \infty$, de modo a manter $p$ finito. Resta então que $\nabla^{2} \vec{u}-\nabla p=0$, que é a equação (1). No contexto do artigo de Stokes, como já dissemos, esta condição surge quando o meio em equilíbrio é o éter luminífero. 


$$
\begin{aligned}
& -\frac{d p}{d x}+\frac{d^{2} \alpha}{d x^{2}}+\frac{d^{2} \alpha}{d y^{2}}+\frac{d^{2} \alpha}{d z^{2}}=0 \\
& -\frac{d p}{d y}+\frac{d^{2} \beta}{d x^{2}}+\frac{d^{2} \beta}{d y^{2}}+\frac{d^{2} \beta}{d z^{2}}=0 \\
& -\frac{d p}{d z}+\frac{d^{2} \gamma}{d x^{2}}+\frac{d^{2} \gamma}{d y^{2}}+\frac{d^{2} \gamma}{d z^{2}}=0 \\
& p=-\kappa\left(\frac{d \alpha}{d x}+\frac{d \beta}{d y}+\frac{d \gamma}{d z}\right)
\end{aligned}
$$

No caso limite ideal em que o sólido é incompressível, $\kappa$ terá um valor infinito e teremos a relação

$$
\frac{d \alpha}{d x}+\frac{d \beta}{d y}+\frac{d \gamma}{d z}=0
$$

As equações (1) e (2) expressam as condições de equilíbrio no interior de um solido elástico incompressível. Estas equações serão empregadas para a representação de forças nos vários problemas físicos considerados neste artigo.

A equação (1) mostra apenas que a expressão

$$
\nabla^{2} \alpha d x+\nabla^{2} \beta d y+\nabla^{2} \gamma d z
$$

(na qual $\nabla^{2}$ denota a operação $d^{2} / d x^{2}+d^{2} / d y^{2}+$ $\left.d^{2} / d z^{2}\right)$ tem que ser um diferencial completo e que, portanto, qualquer expressão para $\alpha, \beta, \gamma$, sujeita a esta condição, e que satisfaça (2), irá representar o estado interno do corpo, produzido pela ação de forças em sua superfície ou superfícies limítrofes.

Podemos obter uma solução particular assumindo que 4

$$
\alpha d x+\beta d y+\gamma d z
$$

seja um diferencial completo. Ainda, supondo que tal expressão não é um diferencial completo, podemos assumir que

$$
\left(\frac{d \beta}{d z}-\frac{d \gamma}{d y}\right) d x+\left(\frac{d \gamma}{d x}-\frac{d \alpha}{d z}\right) d y+\left(\frac{d \alpha}{d y}-\frac{d \beta}{d x}\right) d z(\mathrm{c})
$$

seja um diferencial completo e encontrar outra solução. Finalmente, podemos obter uma solução particular por meio de uma terceira suposição, de acordo com a qual nenhuma das expressões acima seja um diferencial completo. Irei considerar em seguida estas três soluções, com referência à representação das forças Elétrica, Magnética e Galvânica 5

\footnotetext{
${ }^{4}$ N.T.: No original a condição (b) não foi identificada. Tomamos aqui a liberdade de destacá-la.

${ }^{5}$ N.E.: Por força magnética entende-se, aqui, aquela exercida por imãs; enquanto que a força galvânica é aquela exercida por uma corrente elétrica (que, bem sabemos, tem natureza magnética).
}

\section{i. - Forças Elétricas.}

Seja

$$
r^{2}=x^{2}+y^{2}+z^{2}
$$

e assumamos que

$$
\alpha d x+\beta d y+\gamma d z=-d\left(\frac{1}{r}\right) .
$$

Então, como

$$
\frac{d^{2}}{d x^{2}} \frac{1}{r}+\frac{d^{2}}{d y^{2}} \frac{1}{r}+\frac{d^{2}}{d z^{2}} \frac{1}{r}=0,
$$

a equação (1) estará satisfeita, e os coeficientes do diferencial exato em (a) se anulam, de modo que todas as condições de equilíbrio são satisfeitas ${ }^{6}$ Ora, $1 / r$ é o potencial, em $(x, y, z)$, devido a uma unidade de eletricidade na origem, e

$$
\alpha=\frac{x}{r^{3}}, \quad \beta=\frac{y}{r^{3}}, \quad \gamma=\frac{z}{r^{3}},
$$

são componentes da força exercida no ponto $(x, y, z)]^{7}$

\section{ii. - Forças Magnéticas.}

Seja

$$
\begin{gathered}
\left(\frac{d \beta}{d z}-\frac{d \gamma}{d y}\right) d x+ \\
\left.+\frac{d \gamma}{d x}-\frac{d \alpha}{d z}\right) d y+\left(\frac{d \alpha}{d y}-\frac{d \beta}{d x}\right) d z= \\
=d \frac{l x+m y+n z}{r^{3}} .
\end{gathered}
$$

Esta equação é satisfeita por

$$
\alpha=\frac{m z-n y}{r^{3}}, \beta=\frac{n x-l z}{r^{3}}, \gamma=\frac{l y-m x}{r^{3}} \text {, }
$$

${ }^{6}$ N.E.: É, portanto, uma configuração com $p$ constante. ${ }^{7}$ N.E.: A analogia aqui estabelecida é entre o vetor deformação, $\vec{u}=(\alpha, \beta, \gamma)$, e o campo eletrostático, $\vec{E}$, devido a uma carga puntual unitária na origem. Note porém, que Thomson se referiu a este, em suas considerações introdutórias, como uma atração e portanto a correspondência é, a rigor, $\vec{u} \leftrightarrow-\vec{E}$. O leitor irá notar que, em todos os casos apresentados, os sinais dos correspondentes campos vetoriais estão invertidos. Não se trata, meramente, de uma convenção contrária à adotada nos dias atuais. Trata-se da interpretação física dos campos neste contexto. Neste caso, por exemplo, se o meio é esticado para fora de um centro, radialmente, sua tendência é a de retornar a condição não esticada. O efeito sobre um ponto a uma distância do centro é, então, o de uma "atração" para o centro. Considerações similares se aplicam aos outros casos. 
que também satisfaz a equação (2) e faz com que os coeficientes dos diferenciais em (a) se anulem 8 Consequentemente, deslocamentos expressos desta forma podem ser produzidos por forças aplicadas externamente. Ora

$$
\frac{l x+m y+n z}{r^{3}}
$$

é o potencial devido a um pequeno imã, do qual o "momento" é unitário, colocado na origem, com seus eixos de polarização nas direções $l: m: n$ 9 Os componentes $X, Y, Z$ da força que este imã exerce, sobre uma unidade ideal de magnetismo (a terminação de uma fina barra uniformemente

${ }^{8}$ N.E.: Novamente uma configuração de $p$ constante. A correspondência aqui estabelecida é entre o vetor deformação, $\vec{u}=(\alpha, \beta, \gamma)$, e o potencial vetor $\vec{A}=\left(\mu_{0} / 4 \pi\right) \vec{m} \times \vec{r} / r^{3}$ devido a um dipolo magnético puntual na origem, sendo que $\hat{n}=(l, m, n) \leftrightarrow \mu_{0} \vec{m} / 4 \pi$, em unidades do sistema internacional. Usualmente o potencial vetor é atribuído a Maxwell que irá introduzí-lo, em 1856, no seu On Faraday's lines of force 34 como expressão matemática do que Faraday chamava estado eletrotônico e chamado, por Maxwell, função eletrotônica. Maxwell julgava seu o mérito de tê-lo associado à força eletromotriz na indução eletromagnética, mas reconheceria textualmente, no seu "On Faraday's lines", que aprendera sobre a possibilidade de descrever os fenômenos magnéticos a partir de um potencial com orientação e sentido pela leitura do presente artigo de Thomson. O assunto da autoria do potencial vetor é, em nossa opinião, até certo ponto, uma questão de convenção. Maxwell jamais o compreendeu, em seu sentido final, como um campo de calibre. Franz Neumann, em sua memória à academia de Berlim, Die mathematische Gesetze der inducirten elektrischen Ströme, já havia mostrado, em 1845, que a indução eletromagnética de uma corrente sobre outra dependia da quantidade $U=i i^{\prime} \oint_{c} \oint_{c^{\prime}} d \vec{l} \cdot d \overrightarrow{l^{\prime}} / r$, a hoje chamada fórmula de Neumann, onde o potencial vetor do circuito $c^{\prime}, \vec{A}=i^{\prime} \oint_{c^{\prime}} d \overrightarrow{l^{\prime}} / r$, aparece de forma latente, embora não tenha lhe dado, aparentemente, nenhum significado distintivo. No presente artigo Thomson é levado a estabelecer a conexão entre a deformação rotacional do sólido, $\nabla \times \vec{u}$, e os fenômenos magnéticos, provavelmente tendo o efeito Faraday na mente. Embora antecipando-se a Maxwell na abordagem do magnetismo a partir de um potencial vetorial, como em (4), Thomson também não dá nenhum destaque especial a esta grandeza. Em seu belo "Electrodynamics from Ampère to Einstein", no entanto, Darrigol, 7], referindo-se ao presente resultado de Thomson, sentencia: "assim nasceu o potencial vetor". Sobre a consolidação da ideia do potencial vetor como um campo de calibre ver Jackson, 35. Durante a feitura do presente trabalho também tomamos conhecimento da tese de Pereira, A.G., 36, onde uma discussão ampla do surgimento e do significado físico do potencial vetor é realizada.

${ }^{9}$ N.E.: O potencial escalar magnético associado a um dipolo $\hat{n}=(l, m, n)$ na origem, $\psi=\hat{n} \cdot \vec{r} / r^{3}$, surgiu originalmente na mémoire de Poisson, La Théorie du magnétisme, de 1824 , que usara as ideias de fluido magnético, de Coulomb, como a fonte do magnetismo. magnetizada), no ponto $(x, y, z)$, são os coeficientes diferenciais desta expressão, de modo que

$$
X=\frac{d \beta}{d z}-\frac{d \gamma}{d y}, Y=\frac{d \gamma}{d x}-\frac{d \alpha}{d z}, Z=\frac{d \alpha}{d y}-\frac{d \beta}{d x} .
$$

As metades das expressões $(d \beta / d z-d \gamma / d y)$, etc. indicam os componentes das linhas fechadas, paralelas ao eixo, devidas a rotação infinitamente pequena que um elemento do sólido sofre, excetuando-se sua mudança de forma, quando $\alpha d x+\beta d y+\gamma d z$ não é um diferencial completo. Esta rotação, portanto, representa a força magnética resultante, em direção e magnitude 10 .

\section{iii. - Forças Galvânicas.}

Seja agora

$$
\nabla^{2} \alpha d x+\nabla^{2} \beta d y+\nabla^{2} \gamma d z=-d \frac{l x+m y+n z}{r^{3}},
$$

a qual é verdadeira se

$$
\left.\begin{array}{l}
\alpha=\frac{1}{2} \frac{d}{d x} \frac{l x+m y+n z}{r}-\frac{l}{r} \\
\beta=\frac{1}{2} \frac{d}{d y} \frac{l x+m y+n z}{r}-\frac{m}{r} \\
\gamma=\frac{1}{2} \frac{d}{d z} \frac{l x+m y+n z}{r}-\frac{n}{r}
\end{array}\right\} .
$$

Verifica-se prontamente que esta expressão também satistaz a equação (2), e portanto representa um estado interior do corpo, que pode ser produzido por forças aplicadas externamente 11 Encontramos

${ }^{10}$ N.E.:Sendo $\vec{X}=(X, Y, Z)$ e $\hat{n}=(l, m, n)$, então a equação 5 de Thomson é, em notação vetorial, $\vec{X}=-\nabla \times \vec{u}$. Fazendo o cálculo explícito temos então que $\vec{X}=-[3(\hat{n}$. $\hat{r}) \hat{r}-\hat{n}] / r^{3}$, que guarda uma analogia formal com o vetor indução magnética $\vec{B}$ devido a um dipolo magnético $\vec{m}$ na origem. No caso $\vec{X} \leftrightarrow-\vec{B}$, e $\hat{n} \leftrightarrow \mu_{0} \vec{m} / 4 \pi$.

${ }^{11}$ N.E.: É, portanto, uma configuração $\operatorname{com} p=-\hat{n} \cdot \vec{r} / r^{3}$, sendo $\hat{n}=(l, m, n)$. A analogia estabelecida é, novamente, entre o vetor deformação, $\vec{u}=(\alpha, \beta, \gamma)$, e o potencial vetor de um elemento de corrente, $i d \vec{l}$, tal que $\left(\mu_{0} i / 4 \pi\right) d \vec{l} \leftrightarrow-\hat{n}$. A expressão usual deste potencial, quando expresso no calibre de Coulomb, é $d \vec{A}=\left(\mu_{0} i / 4 \pi\right) d \vec{l} / r$. Realizando sobre ele uma transformação de calibre $d \vec{A}^{\prime}=d \vec{A}+\nabla d \alpha$, e tomando $d \alpha=-\left(\mu_{0} i / 8 \pi\right) d \vec{l} \cdot \vec{r} / r$, temos então que $d \overrightarrow{A^{\prime}}$ é o correspondente potencial vetor associado à solução (6) de Thomson. Assim $d \vec{A} \leftrightarrow-\hat{n} / r, d \vec{A}^{\prime} \leftrightarrow \vec{u}$ e $d \alpha \leftrightarrow \hat{n} \cdot \vec{r} / 2 r$. A equação (7) corresponde, portanto, à lei de Biot-Savart $d \vec{B}=\left(\mu_{0} i / 4 \pi\right) d \vec{l} \times \vec{r} / r^{3}$ de modo que $d \vec{B} \leftrightarrow-\nabla \times \vec{u}$. Considerar o elemento de corrente como ente isolado é um artifício para se estabelecer, para o caso de circuitos reais, a lei sobre a qual se deve aplicar o princípio da superposição. É um artifício que remonta à "mémoire" de Ampère (1827) e segundo Greiner, 37, deve ser creditado a Laplace. 
por meio destas equações que

$$
\left.\begin{array}{r}
\frac{d \beta}{d z}-\frac{d \gamma}{d y}=\frac{m z-n y}{r^{3}} \\
\frac{d \gamma}{d x}-\frac{d \alpha}{d z}=\frac{n x-l z}{r^{3}} \\
\frac{d \alpha}{d y}-\frac{d \beta}{d x}=\frac{l y-m x}{r^{3}}
\end{array}\right\},
$$

que são as expressões para os componentes da força devida a um elemento infinitamente pequeno de corrente galvânica, na direção $l, m, n$, na origem, produzida em uma unidade de magnetismo, em um ponto $(x, y, z)$, sendo unitária a intensidade da corrente multiplicada pelo comprimento do elemento. Assim concluímos que a rotação de qualquer elemento do sólido, em um estado expresso por (6), representa a força, em direção e magnitude, de um elemento de fio galvânico.

Eu deveria exceder os presentes limites para entrar no exame especial dos estados de um corpo sólido representando os vários problemas em eletricidade, magnetismo e galvanismo, o que deve ser reservado, portanto, para um artigo futuro.

Glasgow College, 28 Nov., 1846.

\section{Agradecimentos}

Os autores gostariam de agradecer ao prof. Dr. José Maria Filardo Bassalo pelas críticas e sugestões, bem como pelas discussões, sempre acaloradas, provocativas e inspiradoras.

\section{Referências}

[1] L. Pearce Williams, in: Dicionário de Biografias Científicas, editado por César Benjamin (Contraponto Editora Ltda, Rio de Janeiro, 2007), v. I, p. 782-795.

[2] W. Thomson, Cambridge Mathematical Journal 3, 71 (1843).

[3] W. Thomson, Camb. Dub. Math. Journal 1, 75 (1845).

[4] Zed J. Buchwald, in: Dicionário de Biografias Científicas, editado por César Benjamin (Contraponto Editora Ltda, Rio de Janeiro, 2007), v. II, p 12971310 .

[5] S. Thompson, The life of William Thomson (MacMillan and Co., London, 1910).

[6] M. Fadaray, Experimental Researches in Electricity (R. Taylor and W. Francis publ., London, 1855), v.III, p.3.

[7] O. Darrigol, Electrodynamics from Ampère to Einstein (Oxford Univ. Press, Oxford, 2000).
[8] W. Thomson, Proc. Roy Soc. 8, 150 (1857).

[9] J.C. Maxwell, The Scientific Papers of James Clerk Maxwell (Dover Publ. Inc., New York, 1965), v. 1, p. 451-513.

[10] C.W.F. Everitt, in: Dicionário de Biografias Científicas, editado por César Benjamin (Contraponto Editora Ltda, Rio de Janeiro, 2007), v. III, p. 18601893.

[11] J.C. Maxwell, The Scientific Papers of James Clerk Maxwell (Dover Publ. Inc., New York, 1965), v. 1, p. 526-597.

[12] Daniel M. Siegel, Innovation in Maxwell's Electromagnetic Theory (Cambridge Univ. Press, Cambridge, 1991).

[13] B.J. Hunt, The Maxwellians (Cornell University Press, Ithaca and London, 1991).

[14] H. Hertz, Electric Waves (Dover, New York, 1962).

[15] C. Coulomb, Mémoires de l'Académie Royale des Sciences 88, 569, 578, 612 (1785).

[16] Andrè-Marie Ampère, Mémoires de l'Académie Royale des Sciences VI, 175 (1827).

[17] W. Weber, Wilhelm Weber's Werke (Springer, Berlin, 1893), v.3, p. 25.

[18] W. Weber, and R. Kohlrausch, Annalen der Physik und Chemie 99, 10 (1856).

[19] T. Young, Phil. Trans. R. Soc. London 94, 1 (1804).

[20] Fresnel, M.A., Mémoires de l'Académie Royale des Sciences V, 339 (1826).

[21] M.A. Fresnel, Annales de Chimie xvii, 180 (1821).

[22] Robert H. Silliman, in: Dicionário de Biografias $C i$ entificas, editado por César Benjamin (Contraponto Editora Ltda, Rio de Janeiro, 2007), v. I, p. 860-866.

[23] E.T. Whittaker, A History of the Theories of Aether and Electricity: The classical Theories (Thomas Nelson and Sons Ltd., London, 1951).

[24] G. Stokes, Trans. Camb. Phil. Soc. 3, 287 (1845).

[25] M. Faraday, Experimental Researches in Electricity, (R. Taylor and W. Francis publ., London, 1855) v. III, p. 331.

[26] M. Faraday, Experimental Researches in Electricity, (R. Taylor and W. Francis publ., London, 1839) v. I, p. 360 .

[27] M. Faraday, Experimental Researches in Electricity, (R. Taylor and W. Francis publ., London, 1855) v. III, p.1.

[28] M. Faraday, Experimental Researches in Electricity, (R. Taylor and W. Francis publ., London, 1855) v. III, p.3.

[29] M. Faraday, Experimental Researches in Electricity, (R. Taylor and W. Francis publ., London, 1855) v. III, p. 447.

[30] M. Kline, Mathematical Thought From Ancient to Modern Times (Oxford Univ. Press, Oxford, 1972), v. 2.

[31] W. Thomson, The Cambrigde and Dublin Mathematical Journal 2, 61 (1847). 
[32] L.D. Landau and E.M. Lifschiz, Course of Theoretical Physics (Pergamon press, Oxford, 1959), v. 7.

[33] I. Sokolnikoff, Mathematical Theory of Elasticity (McGraw-Hill Book Company, Inc., New York, 1956).

[34] J.C. Maxwell, The Scientific Papers of James Clerk Maxwell (Dover Publ. Inc., New York, 1965), v. 1, p. 155-229.

[35] J.D. Jackson and L.B. Okun, Rev. Mod. Phys 73, 663 (2001).

[36] A.G. Pereira, Um Estudo Histórico da Evolução do Conceito de Potencial Vetor no Eletromagnetismo Clássico. Tese de Mestrado, Universidade de São Paulo, São Carlos, 2009.

[37] W. Greiner, Classical Electrodynamics (SpringerVerlag, New York, 1998). 